\title{
Clasificación de empresas ganaderas doble propósito por calidad y canales de comercialización de la leche en el Caribe colombiano
}

\section{Dual purpose farms classify according quality and milk marketing channels on the colombian Caribbean}

\author{
Elvira Durán-Rojas ${ }^{1}$; Alfonso Calderón-Rangel²*; Javier Ramírez-Montoya ${ }^{3}$ \\ 1Administradora de Empresas, M.Sc., D.Sc. Universidad de Córdoba, Facultad Ciencias de la Salud. Montería - Córdoba, Colombia; e-mail: edrojas@correo. \\ unicordoba.edu.co; (1Dhttps://orcid.org/0000-0002-0811-1704 \\ ${ }^{2}$ MVZ, M.Sc, Ph.D. Universidad de Córdoba, Facultad de Medicina Veterinaria Zootecnia. Montería - Córdoba, Colombia; e-mail: acalderonr@correo. \\ unicordoba.edu.co; Dhttps://orcid.org/0000-0001-6503-8323
}

3Estadístico, M.Sc. Universidad de Córdoba, Departamento de Estadística; e-mail: jaramirez@correo.unicordoba.edu.co; Chttps:// orcid.org/0000-0002-5062-0510

*autor para correspondencia: acalderonr@correo.unicordodoba.edu.co

Cómo citar: Duran-Rojas, E.; Calderón-Rangel, A.; Ramírez-Montoya, J. 2020. Clasificación de empresas ganaderas doble propósito por calidad y canales de comercialización de la leche en el Caribe colombiano. Rev. U.D.C.A Act. \& Div. Cient. 23(2):e1358. http://doi. org/10.31910/rudca.v23.n2.2020.1358

Artículo de acceso abierto publicado por Revista U.D.C.A Actualidad \& Divulgación Científica, bajo una licencia Creative Commons CC BY-NC 4.0

Publicación oficial de la Universidad de Ciencias Aplicadas y Ambientales U.D.C.A, Institución de Educación Superior Acreditada de Alta Calidad por el Ministerio de Educación Nacional.

Recibido: Octubre 20 de 2019 Aceptado: Septiembre 10 de 2020 Editado por: Adriana Posada Arrubla

\section{RESUMEN}

Una leche de excelente calidad debe presentar altos porcentajes de proteína, grasa y sólidos totales, bajos recuentos de mesófilos y de células somáticas y estar libres de inhibidores y sustancias extrañas, para asegurar su inocuidad. El objetivo del presente estudio fue realizar una clasificación de empresas ganaderas de doble propósito, con base a la calidad de la leche y sus canales de comercialización, en una subregión del medio Sinú, en el Caribe colombiano. El tipo de estudio fue descriptivo transversal. Se determinaron variables fisicoquímicas, microbiológicas de la leche y de la sanidad de la ubre, y, por medio de una encuesta, se analizaron parámetros zootécnicos, para establecer factores de manejo. Igualmente, mediante un análisis de componentes principales y correspondencias múltiples, se categorizó la productividad y la competitividad, de acuerdo con los parámetros de calidad de la leche cruda. La mayoría de las empresas ganaderas fueron consideradas de mediana extensión con ordeño manual y almacenamiento de la leche en cantinas. El porcentaje de vacas en ordeño no fue alto y una tercera parte de los productores manifestaron mayores ingresos, por ventas diferentes a la leche. Se determinó que la mayor competitividad, se presentó en las empresas ganaderas que realizan excelentes prácticas de manejo y se asoció con altos índices de calidad y productivos. En consecuencia, es necesario implementar las buenas prácticas ganaderas, para aumentar la competitividad.

Palabras claves: Comercialización; Inocuidad alimentaria; Leche cruda; Células somáticas (NLM). 


\section{ABSTRACT}

An excellent quality milk must have high protein, fat and total solids percentages, low mesophilic and somatic cells counts, and to be free of inhibitor and foreign substances in order to ensure its safety. The objective of this study was to classify the dual-purpose farms based on its milk quality and its marketing channels in a subregion of the middle Sinu in the colombian Caribbean. The study type was descriptive cross sectional. Physicochemical, microbiological variables and udder health were analyzed. Zootechnical parameters were evaluated in order to stablish the manage factors. Equally, through an analysis of mayor compounds and multiple matches the productivity and competitiveness of the farms was categorized based on row milk quality parameters. Most livestock companies were considered as medium length with manual milking and milk storage in canteens. The percentage of cows in milking are not high and a third part of the producer manifested higher income by sales other than milk. It was determined that the higher competitiveness associated with higher quality and productivity indexes occurred in farms where best management practices were implemented. It is necessary to implement good livestock practices to increase the competitiveness.

Keywords: Marketing; Food safety; Raw milk; Somatic cells (NLM).

\section{INTRODUCCION}

En Colombia hay dos sistemas de producción de leche. El sistema de lechería especializada, que se ubica en las zonas de trópico alto $(\geq 1.800 \mathrm{~m}$ s.n.m.), donde hay predomino de las razas Bos taurus, un uso más intensivo de los factores (capital tierra y trabajo), mayor utilización de los suplementos alimenticios y ordeño mecanizado y el doble propósito, que se localiza en zonas de trópico bajo $(\leq 12.000 \mathrm{~m}$ s.n.m.), cuya producción de leche se hace con cruces Bos taurus x Bos indicus taurus, ganaderías de tipo extensiva, por la alta disponibilidad de tierras en estas zonas (Holmann et al. 2003).

Una leche de excelente calidad debe presentar un porcentaje de proteína $>3,2 \%$, grasa $>3,5$, sólidos totales $>12,2$, un bajo número de mesófilos $<50.000 \mathrm{Ufc} / \mathrm{mL}$ y de células somáticas $<100.000 \mathrm{CS} /$ $\mathrm{mL}$ (Calderón et al. 2006), libre de inhibidores y se debe asegurar su inocuidad (Minprotección, 2006). La calidad de la leche depende de las características fisicoquímicas, los estándares de higiene y la calidad nutricional; sin embargo, las prácticas de cría de animales, la recolección y el procesamiento antihigiénico, pueden afectar su calidad. La calidad fisicoquímica y microbiológica de la leche es variable y depende de varios factores: genéticos, fisiológicos, nutricionales, ambientales, características individuales, de manejo, selección, mejoramiento y salud de la glándula mamaria (Vittori et al. 2008; Briñez et al. 2008; Ruiz-Cortés et al. 2012; Martínez-Álvarez et al. 2017; Jamali et al. 2018).

La elaboración de alimentos inocuos y de excelente calidad marca la pauta en la globalización de los sistemas agropecuarios, por lo cual, se debe mantener de manera constante en los diferentes eslabones de la cadena láctea. Las empresas ganaderas que se dedican a la producción de leche son el primer eslabón de la cadena, donde se debe asegurar los recursos necesarios para la obtención de materias primas, de excelente calidad (Calderón et al. 2006).

Se ha indicado que la garantía de la calidad y la seguridad de los productos lácteos es la base y el requisito previo para el desarrollo sostenible y la ventaja competitiva de la industria láctea; para lo cual, el alto rendimiento en el aseguramiento de la calidad, en todas las etapas de la cadena láctea, ayuda a las empresas a ganar la confianza en los consumidores (Ding et al. 2018).

Ante la globalización, el libre mercado y la apertura comercial, se hace inevitable que los productores tengan estrategias de competitividad para usar eficientemente los factores de producción, lo cual, obliga a los diferentes entes de las cadenas productivas a innovar y experimentar cambios constantes, para vencer los paradigmas de la globalización (Burbano et al. 2011). La competitividad, se puede concebir como un sistema multidimensional, porque actúan diferentes niveles y elementos cualitativos y cuantitativos, que participan en su determinación; asimismo, incluye la existencia de diversos enfoques de análisis e indicadores, percibida, a nivel global, como índice de crecimiento económico de un país, que pretende una mayor competitividad, para conquistar nuevos mercados (Saavedra et al. 2014).

La evaluación de la competitividad implica contemplar todos los factores que la generan y su impacto, para la formulación de estrategias frente a los competidores (Barrios \& Olivera, 2013). La implementación de la calidad y prácticas de seguridad ayudan a las empresas de alimentos a mantener su competitividad en el mercado (Kafetzopoulos \& Gotzamani, 2014). Por eso, adoptar la calidad en los alimentos es un sistema de gestión de seguridad alimentaria para los consumidores, que le permite a las empresas obtener ventajas competitivas (Cao et al. 2004).

Colombia firmó varios tratados de libre comercio y se requiere que la producción de leche sea más competitiva, debido a que la producción dejó de ser protegida y este sector enfrenta graves problemas de competitividad (López-Jiménez, 2006; Szegedy-Maszák, 2014); por lo cual, mediante resolución 0017 de 2012 del Ministerio de Agricultura y Desarrollo Rural (MADR, 2012), estableció el pago de la leche por parámetros de calidad y bonificaciones o castigos, sobre los parámetros higiénicos y composicionales.

La globalización y la apertura económica, junto a la política de pago por calidad de la leche, brinda la oportunidad para que los productores de leche conozcan su grado de productividad y así poder implementar acciones, para aumentar su competitividad (Barrios \& Olivera, 2013). En Colombia, el sistema doble propósito ha contribuido alrededor del 60\% de la producción nacional de leche (Carulla \& Ortega, 2016), cuya producción total diaria, a 2018, fue de 20.159.014 litros, donde Córdoba produjo 1.373.543 litros-día, lo que equivale al 7\% de la producción diaria nacional (MADR, 2019).

A pesar que estas leches presentan buenos índices fisicoquímicos tienen deficiencias en la calidad microbiológica y de buenas prácticas 
ganaderas (Rodríguez et al. 2014; Arrieta et al. 2019), sumado a que este sector ganadero, se compone, en su mayoría, de pequeños productores con deficiencia en equipos de ordeño y enfriamiento de unidades lecheras tecnificadas, donde la informalidad es notable en la comercialización de sus productos, lo que afecta en la calidad y en la competitividad de la leche.

El objetivo del presente estudio fue realizar una clasificación de empresas ganaderas del sistema doble propósito, con base en la calidad y sus canales de comercialización, en la subregión del medio Sinú, en el Caribe colombiano, para satisfacer las exigencias del consumidor, incrementar su consumo e impulsar nuevos mercados.

\section{MATERIALES Y MÉTODOS}

Ubicación geográfica. El estudio, se implementó en la región del medio Sinú, del departamento de Córdoba, que pertenece a la región Caribe, enmarcada bajo las siguientes características climáticas: temperatura media $29^{\circ} \mathrm{C}$, precipitación entre $1.500 \mathrm{y}$ $2.000 \mathrm{~mm}$ anuales, humedad relativa del $85 \%$ y 10 a $20 \mathrm{~m}$ s.n.m. (IDEAM, 2001). Con un área de $5.178 \mathrm{~km}^{2}$ y clasificada dentro de un bosque seco tropical, cobertura boscosa, sus pastos sustituyen toda vegetación y sus suelos son profundos, aptos para cultivos transitorios (CVS, 2015).

Tipo de estudio y muestreo. Se implementó un estudio descriptivo de corte transversal con muestreo no probabilístico; se seleccionaron empresas ganaderas del sistema doble propósito en la subregión del medio Sinú, Córdoba, Colombia (Figura 1).
Toma de las muestras de leche. Como en la gran mayoría de las empresas ganaderas, la leche se almacenó en cantinas u otros recipientes de plástico hasta la entrega a los transportadores; de cada uno de estos recipientes, por medio de un agitador manual de acero inoxidable estéril, se mezcló durante cinco minutos y con un cucharón de acero inoxidable estéril, se tomó una submuestra de $25 \mathrm{~mL}$; todas las submuestras de un mismo proveedor se mezclaron y de éstas, se tomó una muestra de leche de $200 \mathrm{~mL}$. En las fincas donde se almacenó en tanque refrigerado, previa agitación por diez minutos, se tomó una muestra $200 \mathrm{~mL}$ de leche, por medio de un cucharón de acero inoxidable estéril (Rodríguez et al. 2014). Todas las muestras, se recolectaron en frascos estériles tapa rosca azul, previamente rotulado, que se conservaron en refrigeración $\left(4^{\circ} \mathrm{C}\right)$ hasta su procesamiento en el Laboratorio de Lactología, de la Facultad de Medicina Veterinaria y Zootecnia de la Universidad de Córdoba. Las muestras, se procesaron en menos de veinticuatro horas después de su recolección. En cada una de las empresas, se levantó una encuesta estructurada, donde se preguntaron características productivas, de manejo y socioeconómicas entre otras (Anexo 1).

Análisis de laboratorio. El porcentaje de grasa (\% grasa), se valoró por medio del método de Gerber; el porcentaje de proteína ( $\%$ proteína), por un analizador ultrasónico de leche (Biolac 60); el de sólidos no grasos (\% SNG), se estimó por el refractómetro de Bertuczi; sólidos totales ( $\%$ ST), por el método de la estufa; recuento de mesófilos aerobios, por el recuento estándar en placa (RSP) y el recuento de células somáticas (RCS), mediante la determinación fluorescente, con el uso de un contador óptico de células, que emplea yoduro de propidio (Rodríguez et al. 2014).

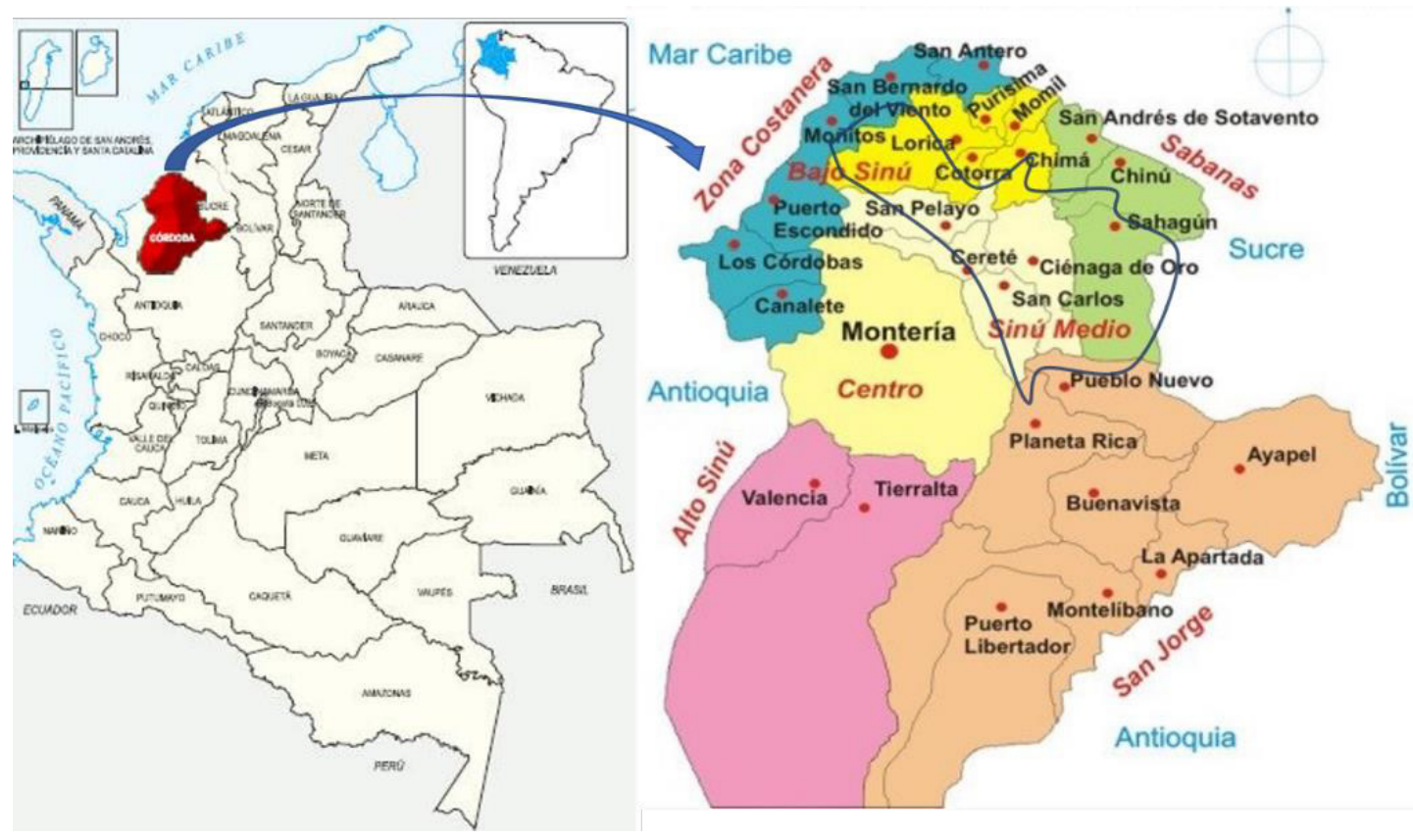

Figura 1. Demarcación de la zona de estudio en una subregión del departamento de Córdoba, Colombia. 
Análisis estadístico. Con los datos recolectados en la encuesta, se adelantó un análisis descriptivo. Seguidamente, se realizó un análisis de componentes principales (ACP) y análisis de correspondencias múltiples (ACM); además, se estimaron modelos lineales generalizados como mixtos y análisis de covarianza. Se confirmaron los resultados de factores significativos, mediante el uso de pruebas no paramétricas; asimismo, se efectuó un análisis de clúster, para determinar las agrupaciones, en las que se clasificaron las empresas ganaderas. Los procedimientos fueron realizados mediante el software estadístico $\mathrm{R}$ versión 3.4.3. Finalmente, se determinó la calidad de la leche, para calcular su precio, de acuerdo con lo establecido en la Resolución 017 de 2012, sobre el sistema de pago al productor; precio que se calculó sobre el valor del dólar en Colombia, en ese momento (TRM=\$1.885).

Confidencialidad. Toda la información recolectada de las empresas ganaderas, se manejó bajo estricta confidencialidad y se contó con la aprobación y colaboración de los propietarios.

\section{RESULTADOS Y DISCUSIÓN}

Se seleccionaron once empresas ganaderas del sistema doble propósito, las cuales, poseen un sistema de producción muy similar a otras regiones tropicales de América, donde sus ingresos económicos se obtienen por la venta de leche de vacas de cruces Bos indicus y Bos Taurus, ordeñadas, principalmente, en forma manual y con el ternero, para estimular el descenso de la leche y la venta de carne, a partir de becerros destetados y de vacas. La carga animal fue muy variada y la alimentación es por pastoreo extensivo en praderas, con pastos nativos e introducidos y un nivel de suplementación variable (DANE, 2015).

La actividad pecuaria de estas once empresas ganaderas fue el doble propósito (producción de carne y leche); el $91 \%$ tuvo como principal actividad la producción de leche, excepto, una empresa que hace la fase de ceba; sin embargo, el 36\% de los productores recibieron más recursos económicos por venta de terneros destetos, lo que representa un ingreso al sistema (Botero \& Rodríguez, 2006) y permite una mayor flexibilidad a este sistema de producción.

Las vacas en ordeño constituyeron en promedio el 59\% del total de las vacas y el $41 \%$ de las vacas secas. Se ha reportado una baja eficiencia en el manejo del recurso animal, cuando la proporción de vacas en ordeño fue de $55,60 \% \pm 13.53$ (Sulbarán et al. 2008), lo que demuestra la importancia de este indicador, debido a la reducción de la producción total y por hectárea de leche (Sánchez et al. 2008). Un parámetro para medir la productividad en una empresa ganadera es la producción por hectárea año, que depende de estos indicadores (Osorio, 2008). La producción promedio de leche-día (445,5 litros) y la producción de leche por vaca (4,9 litros), en el actual estudio, fue baja.

Solo el 27\% de las empresas ganaderas se les reconoció bonificación por enfriamiento, debido a que poseen ordeño mecánico y tanque frío; las restantes empresas $(73 \%)$ realizaron ordeño manual y acopiaron leche en cantinas de aluminio o plástico, recipientes no apropiados que, junto a la alta temperatura ambiental y ausencia de la cadena de frío, disminuyen la calidad microbiológica (Chacón, 2005), por el aumento de los mesófilos, condiciones que pueden generar desconfianza en los consumidores (Ding et al. 2018) y afectar la competitividad en el mercadeo de la leche (Kafetzopoulos \& Gotzamani, 2014).

Por otra parte, las características fisicoquímicas, microbiológicas y células somáticas de las leches en este estudio, se presentan en la tabla 1. Respecto a los resultados obtenidos, existen mejores indicadores de calidad y de producción, reportados en lecherías especializadas, debido al uso más intensivo de los factores de producción (Gamarra, 2004; Barrios \& Olivera, 2013); no obstante, en empresas ganaderas del sistema doble propósito en Galeras (Sucre), presentaron bajos niveles tecnológicos, buena composición fisicoquímica y alta incidencia de mastitis (Botero et al. 2012). De hecho, la mayoría (88\%) de las empresas ganaderas de estudio deben incrementar su productividad (cantidad y calidad) por unidad de producción y así competir con los mismos recursos, en mercados más exigentes.

Se observó que la composición fisicoquímica fue similar, pero se presentaron diferencias en los mesófilos y en células somáticas (Tabla 1). Se logró determinar que la calidad microbiológica de la leche es un factor de competitividad (Herrera et al. 2014). En siete empresas, las leches fueron de excelente calidad microbiológica, porque sus mesófilos fueron $<50 \times 10^{3} \mathrm{Ufc} / \mathrm{ml}$, de acuerdo con lo recomendado (Calderón et al. 2006) y donde los productores debieron recibir la máxima bonificación (US $\$ 0,04$ por litro), según la normatividad vigente (MADR, 2012); en dos empresas, la calidad microbiológica fue buena, porque los mesófilos oscilaron entre $50 \times 10^{3}$ a $100 \times 10^{3} \mathrm{Ufc} / \mathrm{ml}$ y debió reflejarse en una bonificación de US $\$ 0,03$ por litro. Una de las empresas, a pesar de presentar una regular calidad microbiológica y recuento de mesófilos, que osciló entre $100 \times 10^{3}$ y $300 \times 10^{3} \mathrm{Ufc} / \mathrm{ml}$, debió producir una bonificación de US $\$ 0,02$ por litro; por otra parte, una empresa presentó baja calidad microbiológica, cuyo recuento fue de $>300 \times 10^{3} \mathrm{Ufc} / \mathrm{ml}$ y su precio, se pudo disminuir en US\$0,03 por litro. Esta reducción de la calidad microbiológica, se asoció a deficiencias en los procesos de higienización del ordeño y el almacenamiento; también se demostró que, al disminuir el contenido de mesófilos, se debió reflejar como una bonificación en el precio de venta de la leche.

El RCS, no forma parte del esquema de calidad de la leche en Colombia, pero influye en la calidad y en el volumen de leche producida (Cerón-Muñoz et al. 2007; Calderón et al. 2011; Rodríguez et al. 2014) y refleja, de forma directa, infección en las glándulas mamarias (Rodríguez et al. 2014). Cuando se clasificó, de acuerdo con los parámetros establecidos (Calderón et al. 2006), solo en una empresa ganadera se consideró a la leche de excelente calidad $\left(\leq 50 \times 10^{3} \mathrm{CS} / \mathrm{ml}\right)$; de buena calidad, en dos empresas $\left(\leq 140 \times 10^{3} \mathrm{CS} /\right.$ $\mathrm{ml}$ y en las restantes ocho empresas, de regular a mala calidad. Estos altos recuentos de células somáticas hacen necesario la implementación de prácticas de prevención y de control de la mastitis bovina.

En cuanto a la forma de comercializar la leche, en siete empresas lo hicieron con la industria formal, quienes pagan por criterio de calidad, como bonificación por entregar leche fría, certificación 
Tabla 1. Propiedades fisicoquímicas, microbiológicas y sanidad de las ubres en empresas ganaderas del sistema doble propósito, en el medio Sinú, Córdoba (Colombia).

\begin{tabular}{|c|c|c|c|c|c|c|}
\hline Empresa & $\begin{array}{c}\text { Proteína } \\
\mathbf{\%}\end{array}$ & $\begin{array}{c}\text { Grasa } \\
\mathbf{\%}\end{array}$ & $\begin{array}{c}\text { Sólidos no } \\
\text { grasos } \mathbf{0}\end{array}$ & $\begin{array}{c}\text { Sólidos } \\
\text { totales } \mathbf{\%}\end{array}$ & $\begin{array}{c}\text { Mesófilos } \\
\text { Ufc/mL }\end{array}$ & $\begin{array}{c}\text { Células somáticas } \\
\text { Cs } / \mathbf{m L}\end{array}$ \\
\hline 1 & 3,0 & 5,4 & 8,3 & 13,6 & 3690 & 310000 \\
\hline 2 & 3,0 & 4,2 & 8,2 & 12,2 & 12200 & 405000 \\
\hline 3 & 3,0 & 5,0 & 8,4 & 12,7 & 57000 & 630000 \\
\hline 4 & 3,0 & 4,4 & 8,4 & 12,8 & 144000 & 490000 \\
\hline 5 & 3,0 & 4,5 & 8,5 & 12,9 & 6070 & 885000 \\
\hline 6 & 3,0 & 4,8 & 8,5 & 12,8 & $<700000$ & 452000 \\
\hline 7 & 3,2 & 5,0 & 8,8 & 13,1 & 2000 & 506000 \\
\hline 8 & 3,0 & 4,3 & 8,5 & 12,2 & 3000 & 140000 \\
\hline 9 & 3,2 & 4,7 & 8,4 & 13,1 & 56400 & 50000 \\
\hline 10 & 2,9 & 4,7 & 8,1 & 13,1 & 200 & 396000 \\
\hline 11 & 3,0 & 4,3 & 8,5 & 12,2 & 1033 & 140000 \\
\hline
\end{tabular}

libre de brucelosis y tuberculosis. Los promedios del precio pagados a las empresas por la industria formal fueron superiores a los de la industria informal y solo cinco empresas, el valor pagado fue superior al promedio general (Tabla 2). Se muestra que la industria formal reconoció un mejor precio, lo cual, es atractivo para que las empresas ganaderas implementen buenas prácticas de manejo y la comercialización de la leche en la industria formal.

Con el fin de explicar el precio pagado de la leche a las empresas, mediante las características fisicoquímicas, mesófilos, células somáticas y de las buenas prácticas de manejo y ganaderas, los factores que más influyeron fueron: realizar la terapia de la vaca seca $(p v=0,001)$, pago por bonificaciones $(p v=0,004)$, usar tanque frío ( $p v=0,006)$, menor recuento de mesófilos ( $p v<0,01)$, sólidos no grasos $(\mathrm{pv}<0,01)$, mayor porcentaje de grasa y proteína $(\mathrm{pv}<0,01)$ y estar libre de tuberculosis $(\mathrm{pv}=0,036)$.

Por otro lado, se clasificaron las empresas ganaderas en tres grupos, mediante el dendograma (Figura 2): mejor calidad, una empresa ganadera administrada con criterios empresariales; mediana calidad,

Tabla 2. Precio de la leche pagado y por pagar, acorde a los parámetros de calidad en USD del sistema doble propósito, en el medio Sinú, Córdoba (Colombia).

\begin{tabular}{|c|c|c|c|c|}
\hline Empresa & $\begin{array}{c}\text { Forma de } \\
\text { comercialización }\end{array}$ & $\begin{array}{l}\text { Pago al productor } \\
\text { USD }\end{array}$ & $\begin{array}{c}\text { Valor } \% \text { grasa y proteína } \\
\text { USD }\end{array}$ & $\begin{array}{c}\text { Sólidos totales } \\
\text { USD }\end{array}$ \\
\hline 1 & \multirow{7}{*}{$\begin{array}{l}\text { Industria } \\
\text { formal }\end{array}$} & 0,47 & 0,48 & 0,50 \\
\hline 2 & & 0,44 & 0,43 & 0,44 \\
\hline 4 & & 0,40 & 0,41 & 0,42 \\
\hline 4 & & 0,45 & 0,42 & 0,44 \\
\hline 5 & & 0,42 & 0,44 & 0,46 \\
\hline 6 & & 0,34 & 0,37 & 0,39 \\
\hline 7 & & 0,36 & 0,45 & 0,45 \\
\hline & Prom ind. formal & 0,41 & 0,43 & 0,44 \\
\hline 8 & \multirow{4}{*}{$\begin{array}{l}\text { Industria } \\
\text { informal }\end{array}$} & 0,38 & 0,42 & 0,43 \\
\hline 9 & & 0,32 & 0,43 & 0,44 \\
\hline 10 & & 0,34 & 0,42 & 0,46 \\
\hline \multirow[t]{3}{*}{11} & & 0,37 & 0,41 & 0,42 \\
\hline & Prom ind. informal & 0,35 & 0,42 & 0,44 \\
\hline & Promedio total & 0,39 & 0,43 & 0,44 \\
\hline
\end{tabular}


dos empresas ganaderas con menores criterios empresariales y de baja calidad, ocho empresas, donde el manejo fue tradicional y no hay criterios técnicos para la toma de decisiones. Se ha demostrado que la intensidad y el nivel de producción es asociado a una alta tecnificación (Sulbarán et al. 2008; Vargas-Leitón et al. 2013).

Esta categorización indicó una gran variabilidad del sistema doble propósito. En México, al mejorar el nivel tecnológico no hubo un efecto significativo sobre el rendimiento de las empresas ganaderas
(Vargas-Leitón et al. 2013); sin embargo, en el Cesar (Colombia), se determinó que la capacidad tecnológica y productiva fueron las variables que más influyeron en la fabricación de productos y derivados lácteos (Parody et al. 2016). De igual forma, en Santa Rosa de Osos, Risaralda (Colombia), la temperatura adecuada de enfriamiento de la leche, limpieza y desinfección de equipos, condiciones de higiene durante el ordeño, hizo que las empresas presentaran un adecuado desempeño productivo (Marín et al. 2017).

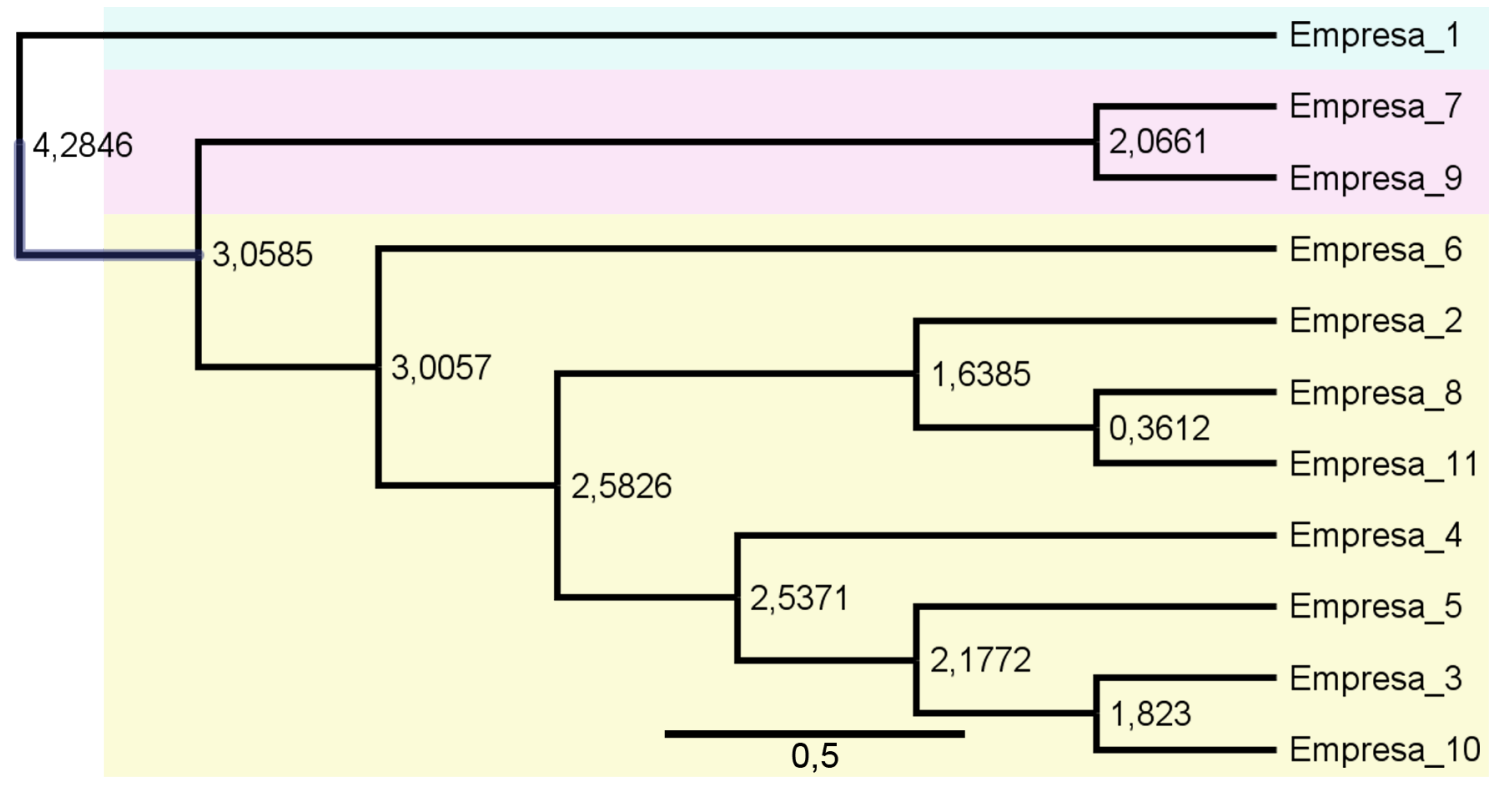

Figura 2. Dendograma que muestra la clasificación de las empresas ganaderas en tres grupos. Azul: mejor calidad; rosado: mediana calidad; amarillo: baja calidad.

De acuerdo con el análisis de componentes principales (Figura 3), se muestra que las elipses del tipo de comprador son diferentes respecto a su dispersión y forma, indicando el efecto que tiene la calidad en las empresas ganaderas. La mejor calidad de la leche, se encuentra en empresas ganaderas donde los compradores son formales. Se destaca la empresa uno, por presentar la mejor calidad de la leche frente a las demás, por su mayor contenido de \%grasa, \%sólidos totales, menor recuento de mesófilos e, igualmente, por la mayor proporción de vacas en ordeño; de igual forma, este mismo comportamiento se observó en estudios previos (Vittori et al. 2008; Briñez et al. 2008; Ruiz-Cortés et al. 2012; Martínez-Álvarez et al. 2017; Jamali et al. 2018).

Por otra parte, cuando se analizaron las características mediante un análisis de correspondencias múltiples (Figura 4), se observa que las empresas ganaderas con más vacas en ordeño, libres de brucelosis y de tuberculosis, con tanque frío, buenas prácticas y comercializan la leche en el mercado formal, presentaron una mejor calidad, que se reflejó en el precio pagado al productor (Cao et al. 2004; MADR, 2012; Kafetzopoulos \& Gotzamani, 2014).

Para finalizar, la metodología presentada permitió categorizar la productividad y competitividad de las empresas ganaderas del sistema doble propósito, de acuerdo con los parámetros fisicoquímicos, microbiológicos, contenido de células somáticas de la leche cruda y las buenas prácticas ganaderas; por tanto, se hace necesario su implementación, mejorar la infraestructura, como uso de tanques de enfriamiento y equipos de ordeño, capacitación y cumplimiento de los criterios de calidad, para mejorar la competitividad de todas las empresas ganaderas y acceder a mercados más exigentes.

Conflicto de intereses: Este manuscrito fue preparado y revisado con la participación de todos los autores, quienes declaramos que no existe conflicto de intereses que pongan en riesgo la validez de los resultados presentados. 


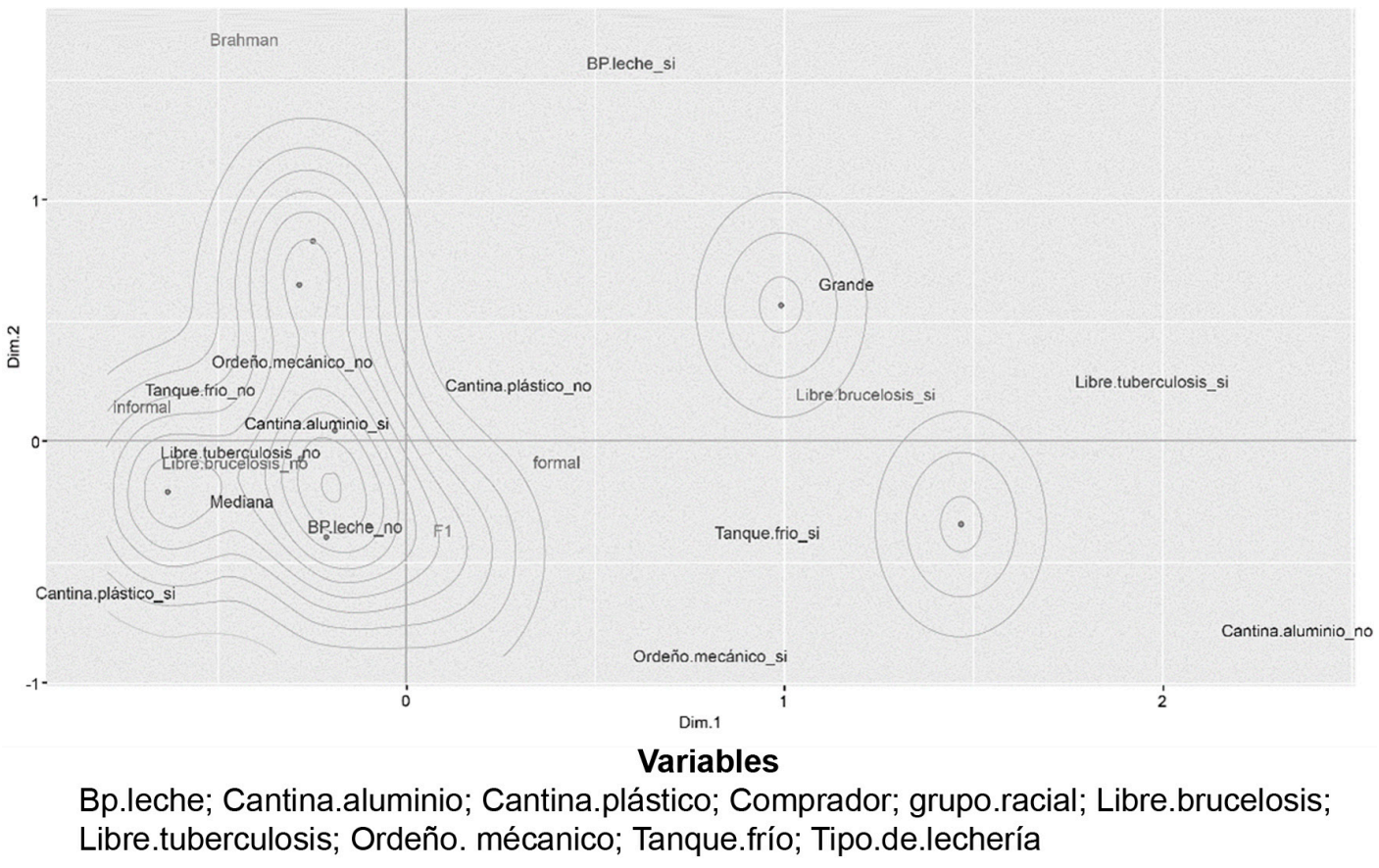

Figura 3. Análisis de componentes principales, donde se observa que las elipses (tipo de comprador) son diferentes y que la mejor calidad se presenta con los compradores formales.

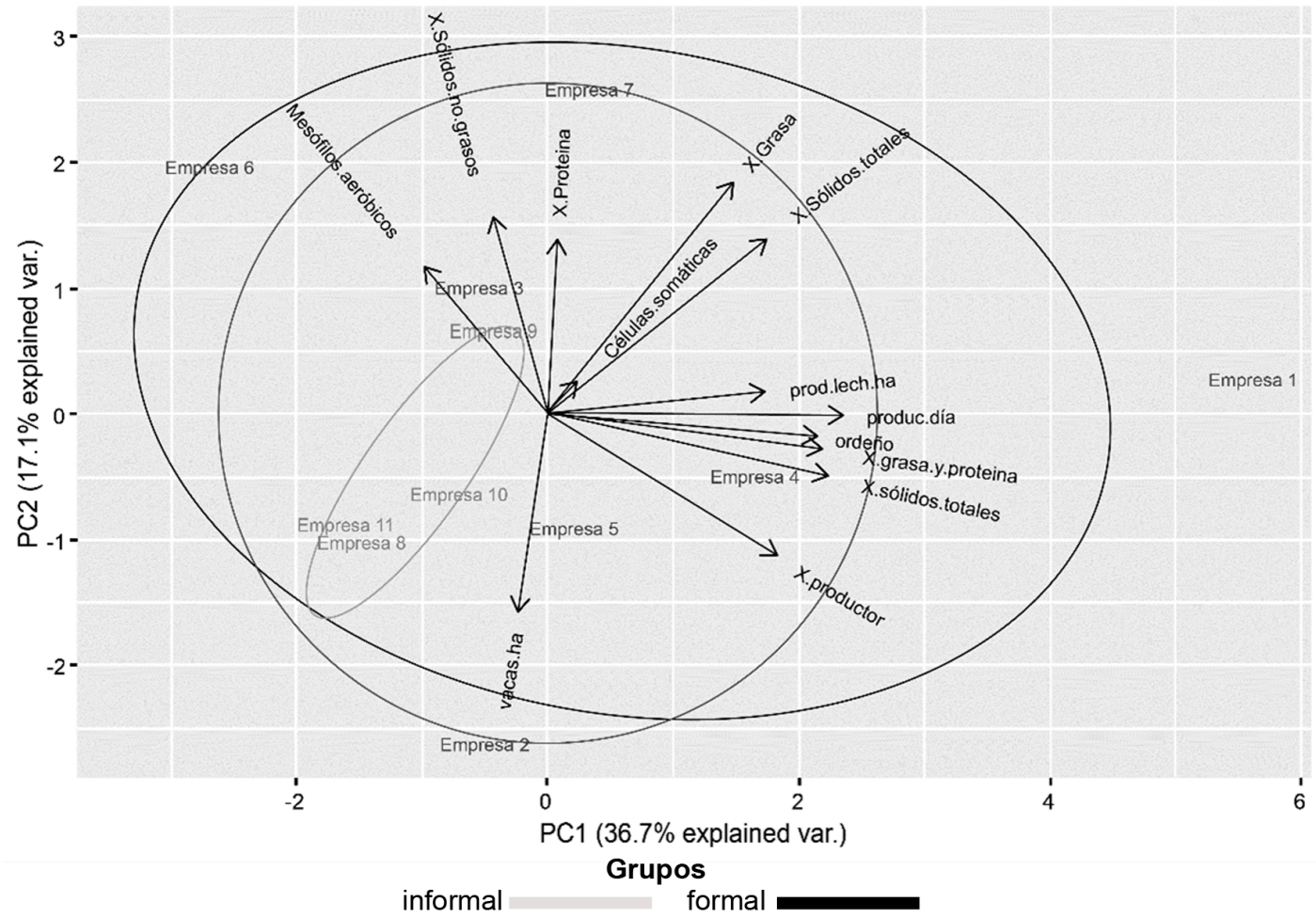

Figura 4. Análisis de correspondencias múltiples, que muestra algunas características de manejo y de producción, relacionadas con los compradores formales de leche cruda. 


\section{REFERENCIAS}

1. ARRIETA, B.G.; GOMEZCACERES, P.L.; ALBIS, F.D.; CALDERÓN-RANGEL, A.; RODRÍGUEZ, R.V. 2019. Calidad de la leche cruda para consumo humano en dos localidades de Sucre, Colombia. Rev MVZ Cordoba. 24(3):7355-7361.

https://doi.org/10.21897/rmvz.1829

2. BARRIOS, H.D.; OLIVERA, M. 2013. Análisis de la competitividad del sector lechero: Caso aplicado al norte de Antioquia, Colombia. Innovar. 23:33-42.

3. BOTERO, L.; RODRÍGUEZ, M. 2006. Costo de producción de un litro de leche en una ganadería del sistema doble propósito, Magangué, Bolívar. Rev MVZ Córdoba. 11(2):806-815.

https://doi.org/10.21897/rmvz.444

4. BOTERO, L.A.; VERTEL, M.M.; FLOREZ, M.L.; MEDINA, J. 2012. Calidad composicional e higiénico-sanitaria de leche cruda entregada en época seca por productores de Galeras, Sucre. Vitae. 19(Supl. 1): 314-316.

5. BRIÑEZ, W.J.; VALBUENA, E.; CASTRO, G.; TOVAR, A.; RUIZ-RAMÍREZ. 2008. Algunos parámetros de composición y calidad en leche cruda de vacas doble propósito en el municipio Machiques de Perijá. Estado Zulia, Venezuela. Revista Científica, FCV-LUZ. 18(5):607-17.

6. BURBANO, E.; GONZÁLEZ, V.; MORENO, E. 2011. La competitividad como elemento esencial para el desarrollo de las regiones. Una mirada al Valle del Cauca. Gestión y Desarrollo. 8(1):51-78.

7. CALDERÓN, A.; ARTEAGA, M.R.; RODRÍGUEZ, V.; ARRIETA, G.J.; BERMÚDEZ, D.C.; VILLAREAL, V.P. 2011. Efecto de la mastitis subclínica sobre el rendimiento en la fabricación del queso costeño. Biosalud. 10(2):16-27.

8. CALDERÓN, A.; GARCÍA, F.; MARTINEZ, G. 2006. Indicadores de calidad de leches crudas en diferentes regiones de Colombia. Revista MVZ-Córdoba. 11(1):725-37. https://doi.org/10.21897/rmvz.457

9. CAO, K.; MAURER, O.; SCRIMGEOUR, F.; DRAKE, C. 2004. The economics of HACCP (Hazard Analysis and Critical Control Points): A literature review. Agribusiness perspectives papers. 7:64.

10. CARULLA, J.E.; ORTEGA, E. 2016. Sistemas de producción lechera en Colombia: Retos y oportunidades. Arch Latinoam Prod Anim. 24(2):83-87.

11. CERÓN-MUÑOZ, M.; AGUDELO, M.; MALDONADOESTRADA, J.G. 2007. Relación entre el recuento de células somáticas individual o en tanque de leche y la prueba CMT en dos fincas lecheras del departamento de Antioquia (Colombia). Rev Col Cienc Pec. 20:472- 483.

12. CORPORACIÓN AUTÓNOMA REGIONAL DE LOS VALLES DEL SINÚ Y SAN JORGE, CVS. 2015. Cobertura geográfica: generalidades del departamento de Córdoba. Disponible desde Internet en:

http: / / www.cvs.gov.co/index.php?option $=$ com content\&view $=$ article $\& i d=149 \&$ Itemid $=160 \&$ lang $=$ es (con acceso el 15/11/2018)

13. CHACÓN, V.A. 2005. Comparación de la titulación de la acidez de leche caprina y bovina con hidróxido de sodio y cal común saturada. Agron. Mesoam. 17(1):55.

https://doi.org/10.15517/am.v17i1.5066

14. DEPARTAMENTO ADMINISTRATIVO NACIONAL DE ESTADÍSTICA, DANE. 2015. La ganadería bovina de doble propósito, una actividad productiva sostenible bajo las buenas prácticas ganaderas (BPGs). Boletín mensual INSUMOS Y FACTORES ASOCIADOS A LA PRODUCCIÓN AGROPECUARIA. 34 tomado de: https://www.dane.gov.co/files/investigaciones/ agropecuario/sipsa/Bol_Insumos31_abr_2015.pdf (con acceso el 15/11/2018).

15. DING, H.; FU, Y.; ZHENG, L.; YAN, Z. 2018. Determinants of the competitive advantage of dairy supply chains: Evidence from the Chinese dairy industry. Int J Prod Econ. 29(2019):360-373. https://doi.org/10.1016/j.ijpe.2018.02.013

16. GAMARRA, J. 2004. Eficiencia técnica relativa de la ganadería doble propósito en la Costa Caribe. Documentos de trabajo sobre Economía regional, Banco de la República (Colombia), (53). Disponible desde Internet en: http://www.banrep.gov.co/sites/default/files / publicaciones/archivos/DTSER-53.pdf (con acceso el 15/11/2018).

17. HOLMANN, F.; RIVAS, L.; CARULLA, J.; RIVERA, B.; GIRALDO, L.A.; GUZMAN, S.; MARTINEZ, M.; MEDINA, A.; FARROW, A. 2003. Evolution of milk production systems in tropical Latin America and its interrelationship with markets: An Analysis of the Colombian case. Livestock Res Rur Dev. 15(9):68.

18. HERRERA, B.; TAMAYO, G.; RODRÍGUEZ, L.; VASCO, C.; ESCOBAR, J.A. 2014. Efecto de la aplicación del sistema de gestión de calidad en la cadena productiva de leche. El Caso de Tuntataco, Chimborazo. Revista Amazónica Ciencia y Tecnología. 3(2):130-139.

19. INSTITUTO DE HIDROLOGÍA METEOROLOGÍA Y ESTUDIOS AMBIENTALES, IDEAM. 2001. La 
atmósfera, el tiempo y el clima. El medio ambiente en Colombia. Disponible desde Internet en:

http://documentacion.ideam.gov.co/openbiblio/ bvirtual/005192/medioambiente/cap3parteI.pdf (con acceso el 15/11/2018).

20. JAMALI, H.; BARKEMA H.W.; JACQUES, M.; LAVALLÉEBOURGET, E.M.; MALOUIN, F.; SAINI, V.; STRYHN, H.; DUFOUR, S. 2018. Invited review: Incidence, risk factors, and effects of clinical mastitis recurrence in dairy cows. J Dairy Sci. 101(6):4729-46. https://doi.org/10.3168/jds.2017-13730

21. KAFETZOPOULOS, D.P.; GOTZAMANI, K.D. 2014. Critical factors, food quality management and organizational performance. Food Control. 40:1-11. https://doi.org/10.1016/j.foodcont.2013.11.029

22. LÓPEZ-JIMENEZ, F. 2006. La competitividad en Colombia: Apertura económica, instituciones de apoyo y seguridad democrática. Revista Universidad Eafit. 42(142):9-25.

23. MARÍN, A.L.; ARREDONDO, B.J.; HERNANDEZ, H.D. 2017. Buenas prácticas ganaderas en hatos lecheros de Santa Rosa de Cabal, Risaralda, Colombia. Rev Colombiana Cienc Anim. 9(Supl):67-75.

https://doi.org/10.24188/recia.v9.nS.2017.523

24. MARTÍNEZ-ÁLVAREZ, M.; RIBOT-ENRÍQUEZ, A.; MARTÍNEZ-VASALLO, A.; CAPDEVILA-VARELA, J.; HERNÁNDEZ-RODRÍGUEZ, R. 2017. Influencia de la época del año sobre la calidad fisicoquímica de la leche en una provincia de la región occidental de Cuba. Rev. Salud Anim. 39(3):1-5.

25. MINISTERIO DE AGRICULTURA Y DESARROLLO RURAL, MADR. 2012. Resolución Número 000017 por la cual se establece el sistema de pago de la leche cruda al proveedor. 18p. Disponible desde Internet en: https:// www.minagricultura.gov.co/ministerio/direcciones/ Documents/d.angie/Res\%20\%20000017\%20de\%202012. pdf (con acceso el 15/11/2018).

26. MINISTERIO DE AGRICULTURA Y DESARROLLO RURAL, MADR. 2019. Cadena Láctea. Dirección de Cadenas Pecuarias, Pesqueras y Acuícolas. Disponible desde Internet en:

http: / / sioc.minagricultura.gov.co/SICLA / Documentos/2019-03-30\%20Cifras\%20Sectoriales.pdf (con acceso el 25/04/2020).

27. MINISTERIO DE LA PROTECCIÓN SOCIAL, MINPROTECCIÓN. 2006. Decreto número 616 del 28 de febrero de 2006. Por el cual se expide el Reglamento Técnico sobre los requisitos que debe cumplir la leche para el consumo humano que se obtenga, procese, envase, transporte, comercializa, expenda, importe o exporte en el país. Disponible desde Internet en:

https:/ /www.ica.gov.co/getattachment/15425e0f-81fb4111-b215-63e61e9e9130/2006D616.aspx (con acceso el $15 / 11 / 2018)$.

28. OSORIO, F. 2008. Gestión empresarial en fincas lecheras. Revista de Medicina Veterinaria y Zootecnia. 47:33-35.

29. PARODY, M.K.D.; JIMÉNEZ, C.L.M.; MONTERO, P.J.F. 2016. Análisis de los factores internos de competitividad: Caso de las empresas lácteas del Cesar, Colombia. Revista Ciencias Estratégicas. 24(35):199-210.

30. RODRÍGUEZ, R.V.; CALDERÓN, R.A.; VERGARA, G.O. 2014. Calidad de leches crudas en tres empresas acopiadoras en Córdoba. Rev Colombiana Cienc Anim. 6(1):103-115.

31. RUIZ-CORTÉS, T.; OROZCO, S.; RODRÍGUEZ, L.S.; IDÁRRAGA, J.; OLIVERA, M. 2012. Factores que afectan el recuento de UFC en la leche en tanque en hatos lecheros del norte de Antioquia-Colombia. Rev U.D.C.A Act. \& Div. Cient. 15(1):147-155. https://doi.org/10.31910/rudca.v15.n1.2012.812

32. SAAVEDRA, G.M.L.; MILLA, T.S.O.; TAPIA, S.B. 2014. Determinación de la competitividad de la PYME en el nivel micro: El caso de del Distrito Federal, México. Revista FIR. 2(4):38-52

33. SÁNCHEZ, T.; LAMELA, L.; LÓPEZ, O.; BENÍTEZ, M. 2008. Comportamiento productivo de vacas lecheras Mambí de Cuba en una asociación de gramíneas y Leucaena leucocephala cv. Cunningham. Pastos y Forrajes. 31(4):1-1.

34. SULBARÁN, L.; DRESCHER, K.; MARTÍNEZ, N.; COLMENARES, O.; RICCA, R. 2008. Patrón tecnológico del sistema con vacunos de doble propósito en la región colinosa del bosque seco tropical en Venezuela. Zootecnia Trop. 26(2):87-93.

35. SZEGEDY-MASZÁK, I. 2014. Free trade and agricultural public policies for producers of milk in Colombia. Vniversitas. 128:357-81. https://doi.org/10.11144/Javeriana.VJ128.ftap

36. VARGAS-LEITÓN, B.; SOLÍS-GUZMÁN, O.; SÁENZSEGURA, F.; LEÓN-HIDALGO, H. 2013. Caracterización y clasificación de hatos lecheros en Costa Rica mediante análisis multivariado. Agron Mesoam. 24(2):257-75.

37. VITTORI, J.; SCHOCKEN-ITURRINO, R.P.; POIATTI, M.L.; PIGATTO, C. P.; PRISCILA, CHIODA, T.P.; MARTINS, R.C.A.; ROJAS, G.G.; FERREIRA R.A.V. 2008. Qualidade microbiológica de leite UHT caprino: pesquisa de bactérias dos gêneros Staphylococcus, Bacillus e Clostridium. Ciência Rural. 38(3):761-765. 